[4] J. Overgaard, D. G. Gonzalez, M. C. C. M. Hulshof, G. Arcangeli, O Dahl, O. Mella, and S. M. Bentzen, "Hyperthermia as an adjuvant to radiation therapy of recurrent or metastatic malignant melanoma-A multicentre randomized trial by the European Society for Hyperthermic Oncology," Int. J. Hypertherm., vol. 12, pp. 3-20, 1996.

[5] A. Rosen, P. Wallinsky, D. Smith, Y. Shi, Z. Kosman, A. Martinez-Hernandez, H. Rosen, F. Sterzer, D. Mawhinney, A. Presser, J. S. Chou, P. Goth, and G. Lowery, "Percutaneous transluminal microwave balloon angioplasty," IEEE Trans. Microwave Theory Tech., vol. 38, pp. 90-93, Jan. 1990

[6] N. C. Sharp, N. Cronin, I. Feldberg, M. Evans, D. Hodgson, and S. Ellis, "Microwaves for menorrhagia: A new fast technique for endometrial ablation," Lancet, vol. 346, pp. 1003-1004, 1995.

[7] J. C. Lin and Y. J. Wang, "The cap-choke catheter antenna for microwave ablation treatment," IEEE Trans. Biomed. Eng., vol. 43, pp. 657-660, June 1996.

[8] A. Taflove, Computational electrodinamics: The finite-difference timedomain method. London, U.K.: Artech House, 1995.

[9] R. L. Higdon, "Numerical absorbing boundary conditions for the wave equation," Math. Comp., vol. 49, pp. 65-90, 1987.

[10] A. W. Guy, "Analysis of electromagnetic fields induced in biological tissues by thermographic studies on equivalent phantom models," IEEE Trans. Microwave Theory Tech., vol. MTT-19, pp. 205-214, 1971.

\section{Detection of Delayed Gastric Emptying from Electrogastrograms with Support Vector Machine}

\author{
Hualou Liang* and Zhiyue Lin
}

\begin{abstract}
A recent study reported a conventional neural network (NN) approach for the noninvasive diagnosis of delayed gastric emptying from the cutaneous electrogastrograms [1]. Using support vector machine, we show that this relatively new technique can be used for detection of delayed gastric emptying and is in fact able to outdo the conventional NN.
\end{abstract}

Index Terms-Artificial neural networks, electrogastrogram, gastric emptying, spectral analysis, support vector machine.

\section{INTRODUCTION}

Gastric emptying is the commonly used test for the assessment of the digestive process of the stomach. Radioscintigraphy is currently the established gastric emptying test for diagnosis of delayed gastric emptying. In this method, a patient is instructed to digest a meal with radioactive materials and then to stay under a gamma camera for acquiring abdominal images for several hours. Although radioscintigraphy is the gold standard for gastric emptying test, it involves radiation exposure and is considerably expensive. It is, therefore, imperative to develop noninvasive and low-cost methods for diagnosis of delayed gastric emptying.

Artificial neural network has been recently used for the noninvasive diagnosis of delayed gastric emptying from cutaneous electrogastrograms (EGGs) [1]. The advantages of this method are mainly due to

Manuscript received November 10, 1999; revised January 13, 2001. Asterisk indicates corresponding author.

*H. Liang is with the Center for Complex Systems, Florida Atlantic University, 777 Glades Road, P.O. Box 3091, Boca Raton, FL 33431 USA (e-mail liang@walt.ccs.fau.edu).

Z. Lin is with the University of Kansas Medical Center, Department of Medicine, Kansas City, Kansas 66160 USA.

Publisher Item Identifier S 0018-9294(01)03400-0. the noninvasive nature of EGGs and free disturbance of the ongoing activity of the stomach. In spite of its success, it or any conventional neural network (even with regularization or weight decay) still suffers from some problems such as over-fitting which results in low generalization ability. Especially, the irrelevant variables will hurt the performance of neural network. In this paper, a relatively new technique called support vector machine (SVM) [2] is introduced to boost the prediction accuracy of delayed gastric emptying from the EGGs.

SVM is a new promising pattern classification technique proposed recently by Vapnik and co-workers [2], [3]. It is based on the idea of structural risk minimization [2], which shows that the generalization error is bounded by the sum of the training set error and a term depending on the Vapnik-Chervonenkis (VC) dimension [2] of the learning machine. Unlike traditional NNs, which minimize the empirical training error, SVM aims at minimizing the upper bound of the generalization error, thus high generalization performance can be achieved. Moreover, SVMs generalization error is related not to the input dimensionality of the problem, but to the margin with which it separates the data. This explains why SVM can have good performance even in problems with a large number of inputs. This paper is intended to explore the application of SVM for detection of delayed gastric emptying from the EGGs and compare its performance with the conventional neural network.

\section{METHODS}

\section{A. EGG Data Preprocessing}

To allow benchmark comparison, the EGG data used in this study is exactly the same as that in [1]. We here will recapitulate the preprocessing procedure for extracting the input parameters; details can be found in [1].

The EGG is a cutaneous recording of gastric myoelectrical activity using abdominal electrodes. The gastric slow wave may range in frequency from one to nine cycles per minute (cpm). The frequency and propagation of gastric contractions are controlled by the gastric slow wave. The normal frequency of the gastric slow wave is in the $2-4 \mathrm{cpm}$ range in humans. The techniques for recording the EGG and gastric emptying were previously described [5]. It has been shown that the spectral parameters of the EGG provide useful information regarding gastrointestinal motility and symptoms [6]. Therefore, a set of EGG spectral parameters were extracted as the inputs of the SVM.

In the experiments, simultaneous recordings of the EGG and scintigraphic gastric emptying test were made in 152 patients with symptoms suggestive of delayed gastric emptying for $30 \mathrm{~min}$ in the fasting state and for $2 \mathrm{~h}$ after a standard test meal. Running spectral analysis of each 2-min EGG data without overlapping were performed to derive EGG parameters. If the peak power of each 2-min EGG was within 2-4 cpm range, we called it normal. Otherwise we called it gastric dysrhythmia. The frequency at which the EGG power spectrum has a peak power in the range of 0.5-9.0 cpm was defined as the EGG dominant frequency. The power at the corresponding dominant frequency was defined as EGG dominant power. The relative change of EGG dominant powers before and after the test meal was defined as the postprandial increase of the EGG dominant power.

The inputs used in the SVM were five parameters which included the dominant frequency in the fasting state, the dominant frequency in the fed state, the postprandial increase of the EGG dominant power, the percentage of normal 2-4 cpm slow waves in the fed state and the percentage of tachygastria in the fed state. The value of each parameter was normalized between zero and one. 


\section{B. SVM for Classification}

In this section we briefly sketch the ideas behind SVM for classification and refer readers to [2]-[4] for a full description of the technique.

Given the training data $\left\{\left(\mathbf{x}_{i}, y_{i}\right)\right\}_{i=1}^{N}, \mathbf{x}_{i} \in \mathcal{R}^{m}, y_{i} \in\{ \pm 1\}$ for the case of two-class pattern recognition, the SVM first maps $x$ from input data $\mathrm{x}$ into a high dimensional feature space by using a nonlinear mapping $\phi, \mathbf{z}=\phi(\mathbf{x})$. In case of linearly separable data, the SVM then searches for a hyperplane $\mathbf{w}^{\mathrm{T}} \mathbf{z}+b$ in the feature space for which the separation between the positive and negative examples is maximized. The $\mathbf{w}$ for this optimal hyperplane can be written as $\mathbf{w}=\sum_{i=1}^{N} \alpha_{i} y_{i} \mathbf{z}_{i}$, where $\alpha=\left(\alpha_{1}, \cdots, \alpha_{N}\right)$ can be found by solving the following quadratic programming $(\mathrm{QP})$ problem: maximize

$$
\boldsymbol{\alpha}^{\mathrm{T}}-\frac{1}{2} \boldsymbol{\alpha}^{\mathrm{T}} \mathbf{Q} \boldsymbol{\alpha}
$$

subject to

$$
\boldsymbol{\alpha} \geq \mathbf{0}, \quad \boldsymbol{\alpha}^{\mathrm{T}} \mathbf{Y}=0
$$

where $\mathbf{Y}^{\mathrm{T}}=\left(y_{1}, \ldots, y_{N}\right)$ and $\mathbf{Q}$ is a symmetric $N \times N$ matrix with elements $Q_{i j}=y_{i} y_{j} \mathbf{z}_{i}^{\mathrm{T}} \mathbf{z}_{j}$. Notice that $\mathbf{Q}$ is always positive semidefinite and so there is no local optimum for the QP problem. For those $\alpha_{i}$ that are nonzero, the corresponding training examples must lie closest to the margins of decision boundary (by the Kuhn-Tucker theorem [7]), and these examples are called the support vectors $(S V s)$.

To obtain $Q_{i j}$, one does not need to use the mapping $\phi$ to explicitly get $\mathbf{z}_{i}$ and $\mathbf{z}_{j}$. Instead, under certain conditions, these expensive calculations can be reduced significantly by using a suitable kernel function $K$ such that $K\left(\mathbf{x}_{i}, \mathbf{x}_{j}\right)=\mathbf{z}_{i}^{\mathrm{T}} \mathbf{z}_{j}$, and $Q_{i j}$ is then computed as $Q_{i j}=y_{i} y_{j} K\left(\mathbf{x}_{i}, \mathbf{x}_{j}\right)$. By using different kernel functions, the SVM can construct a variety of classifiers, some of which as special cases coincide with classical architectures.

\section{Polynomial classifiers of degree $p$}

$$
K\left(\mathbf{x}_{i}, \mathbf{x}_{j}\right)=\left(\mathbf{x}_{i}^{\mathrm{T}} \mathbf{x}_{j}+1\right)^{p}
$$

\section{Radial-basis function (RBF) classifier}

$$
K\left(\mathbf{x}_{i}, \mathbf{x}_{j}\right)=e^{-\left\|\mathbf{x}_{i}-\mathbf{x}_{j}\right\|^{2} / \sigma} .
$$

\section{Neural networks}

$$
K\left(\mathbf{x}_{i}, \mathbf{x}_{j}\right)=\tanh \left(\kappa \mathbf{x}_{i} \mathbf{x}_{j}+\theta\right) .
$$

In the RBF case (4), the SVM automatically determines the number (how many SVs) and locations (the SVs) of RBF centers and gives excellent result compared to classical RBF [8]. Equation (5) gives a particular kind of two-layer sigmoidal NN. In this case, the first layer consists of $N_{s}$ (the number of SVs) sets of weights, each set consisting of $d$ (the dimension of the data) weights, and the second layer consists of $N_{s}$ weights $\left(\alpha_{i}\right)$. The architecture (the number of weights) is determined by SVM training.

During testing, for a test vector $\mathbf{x} \in \mathcal{R}^{m}$, we first compute

$$
a(\mathbf{x}, \mathbf{w})=\mathbf{w}^{\mathrm{T}} \mathbf{z}+b=\sum_{i} \alpha_{i} y_{i} K\left(\mathbf{x}, \mathbf{x}_{i}\right)+b
$$

and then its class label $o(\mathbf{x}, \mathbf{w})$ is given by

$$
o(\mathbf{x}, \mathbf{w})=\left\{\begin{aligned}
1, & a(\mathbf{x}, \mathbf{w})>0 \\
-1, & \text { otherwise }
\end{aligned}\right.
$$

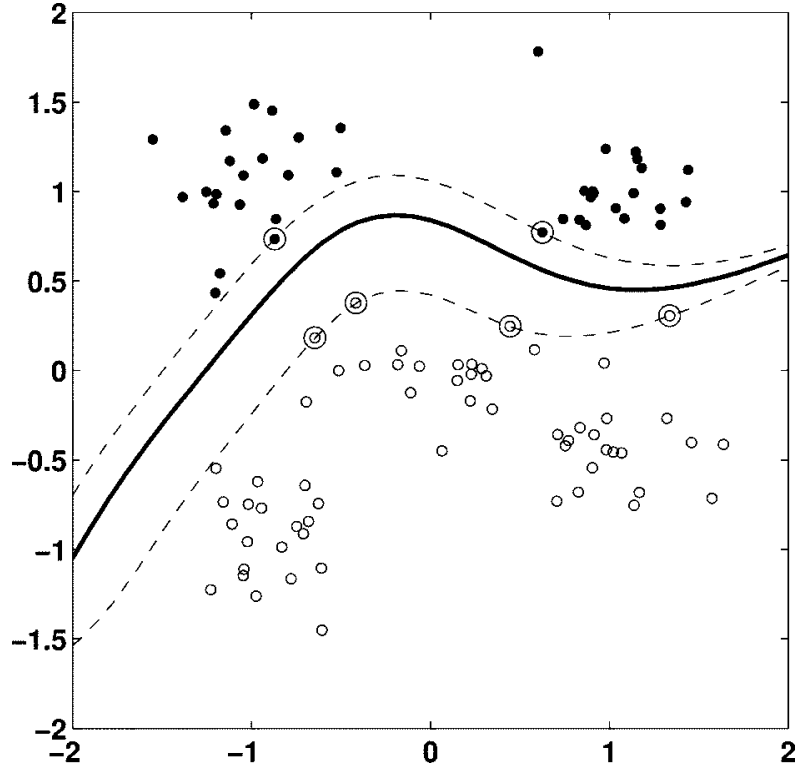

Fig. 1. A simulated two-dimensional classification problem solved by a SVM with a cubic polynomial kernel (3). Circles and disks are two classes of training examples. The solid line is the decision boundary; the two dashed lines are the margins of the decision boundary. The support vectors found by the algorithm (marked by extra circles) are examples which are critical for the given classification task.

The above algorithm for separable data can be generalized to the nonseparable data by introducing nonnegative slack variables $\xi_{i}, i=$ $1, \ldots, N$ [3]. The resultant problem becomes minimizing

$$
\frac{1}{2}\|\mathbf{w}\|^{2}+C \sum_{i=1}^{N} \xi_{i}
$$

subject to

$$
\xi_{i} \geq 0, \quad y_{i} a\left(\mathbf{x}_{i}, \mathbf{w}\right) \geq 1-\xi_{i}, \quad i=1, \ldots, N
$$

Thus, once an error occurs, the corresponding $\xi_{i}$ which measures the (absolute) difference between $a\left(\mathbf{x}_{i}, \mathbf{w}\right)$ and $y_{i}$ must exceed unity, so $\sum_{i} \xi_{i}$ is an upper bound on the number of training error. $C$ is a constant controlling the tradeoff between training error and model complexity. Again, minimization of (8) can be transformed to a QP problem: maximize (1) subject to the constraints $\mathbf{0} \leq \boldsymbol{\alpha} \leq C$ and $\alpha^{\mathrm{T}} \mathbf{Y}=0$. Its convergence behavior is nothing but solving a linearly constrained convex quadratic programming problem.

To provide some insight into how SVM behaves in input space, we show a simple binary toy problem solved by a SVM with a polynomial kernel (3) of degree 3 (see Fig. 1). The support vectors, indicated by extra circles, define the margin of largest separation between the two classes (circles and disks). It was shown [3] that the classification with optimal decision boundary (the solid line) generalizes well as opposed to the other boundaries found by the conventional NNs.

\section{RESULTS}

Based on the scintigraphic gastric emptying test the EGG data obtained from 152 patients were divided into two groups: 76 patients with delayed gastric emptying and 76 patients with normal gastric emptying. $50 \%$ of the EGG data from each group were selected at random as the training set, and the remaining data were used as the testing set. We used the same criteria as [1] to evaluate the performance of the SVM. 
Specifically, the percentages of correct classification $(C C)$, sensitivity $(S E)$ and specificity $(S P)$ were computed as follows:

$$
\begin{aligned}
& C C=100 \times \frac{T P+T N}{N} \\
& S E=100 \times \frac{T P}{T P+F N} \\
& S P=100 \times \frac{T N}{T N+F P}
\end{aligned}
$$

where

$N \quad$ total number of the patients;

TP number of the true positives;

$T N \quad$ number of true negatives;

$F N \quad$ number of false negatives;

FP number of false positives [9].

Results for the three different kernels [polynomial kernels (3), radial-basis function kernels (4), and sigmoid kernels (5)] are summarized in Table I. In all experiments, we used the support vector algorithm with standard quadratic programming techniques and $C=5^{1}$

(8). It can be seen from Table I that the SVM with the radial-basis kernels performs best $(89.5 \%)$ among the three classifiers. In all three cases, the SVMs exhibit higher generalization ability compared to the best performance achieved (85\%) on the same data set with a two-layer NN [1]. The low sensitivity and the high specificity observed in Table I are consistent with the results in [1]. Table I (last column) also shows the numbers of SVs found by different types of support vector classifiers which contains all the information necessary to solve the given classification task.

\section{DISCUSSION AND CONCLUSION}

A SVM approach is proposed for the noninvasive diagnosis of delayed gastric emptying from the cutaneous EGGs. We have shown that, compared to the standard NN technique, the SVM exhibits higher prediction accuracy of delayed gastric emptying.

Radioscintigraphy is currently the gold standard for quantifying gastric emptying. The application of this technique is radioactive and considerably expensive, and usually limited to very sick patients. This motivates ones to develop the low-cost and noninvasive methods based on the EGG. EGG is attractive because of its noninvasiveness (no radiation and no intubation). Once the technique is learned, studies are relatively easy to perform. Unlike radiositigraphy, EGG provides information about gastric myoelectrical activity in both the fasting and postprandial periods [10]. Numerous studies have been performed on the correlation of the EGG and gastric emptying [1], [5], [6], [10]-[14]. Although some of the results are still controversial, it is generally accepted that an abnormal EGG usually predicts delayed gastric emptying [5], [10]. This is because gastric myoelectrical activity modulates gastric motor activity. Abnormalities in this activity may cause gastric hypomotility and/or uncoordinated gastric contractions, yielding delayed gastric emptying. Moreover, the accuracy of the prediction is associated with the selection of EGG parameters and methods of prediction. Previous studies [1], [5], [6] have shown that spectral parameters of the EGG provide useful information regarding gastrointestinal motility and symptoms, whereas the waveform of the EGG is unpredictable and does not provide reliable information. This leaded us to use the spectral parameters of the EGG as the inputs of the SVM. The feature selection of surface EGGs was based on the statistical analysis of the EGG

${ }^{1}$ Changing $c$ while keeping the same number of outliers provides an alternative for the merit measure of the SVM. We use the above criteria which allow us to make direct comparison with previous result.
TABLE I

TESTING REsUlts FOR THE THREE

DifFERENT KeRnels DesCRIBED IN SECTION II WITH THE PARAMETERS In PARENTHESES. The $C C, S E$, AND $S P$ ARe, Respectively, the Percentages of CoRrect Classification, Sensitivity, and SPECIFICITY. THE Numbers of SVS FOUND By DifFERENT ClassifiERS ARE ALSO SHOWN IN THE LAST COLUMN

\begin{tabular}{c|c|c|c|c}
\hline & $\mathrm{CC}(\%)$ & $\mathrm{SE}(\%)$ & $\mathrm{SP}(\%)$ & \# of SVs \\
\hline Polynomial $(p=5)$ & 88.2 & 81.6 & 94.7 & 30 \\
\hline $\mathrm{RBF}(\sigma=0.6)$ & 89.5 & 84.2 & 94.7 & 48 \\
\hline $\mathrm{NN}(\kappa=0.6, \theta=0.9)$ & 85.5 & 79.0 & 92.1 & 42 \\
\hline
\end{tabular}

parameters between the patients with normal and delayed gastric emptying [1], [5], [10]. The size of the training set in this study is equal to that of the testing set. The purpose for using these balanced training and testing set is to conveniently compare with the previous result obtained with the NN [1].

It is important to stress the essential differences between the SVM and NNs. First, the SVM always finds a global solution which is in contrast to the NNs, where many local minima usually exist [4]. Second, the SVM does not minimize the empirical training error alone which NNs usually aim at. Instead it minimizes the sum of an upper bound on the empirical training error and a penalty term that depends on the complexity of the classifier used. Despite the high generalization ability of the SVM the optimal choice of kernel for a given problem is still a research issue.

In summary, the proposed method seems to be a potentially useful tool for the automated diagnosis of delayed gastric emptying. Further research in this field will include adding more EGG parameters as inputs to the SVM to improve the performance.

\section{ACKNOWLEDGMENT}

The authors would like to thank Dr. J. Z. Chen and Dr.R. W. McCallum for their support in data selection.

\section{REFERENCES}

[1] J. D. Z. Chen, Z. Y. Lin, and R. W. McCallum, "Non-invasive featurebased detection of delayed gastric emptying in humans using neural networks," IEEE Trans. Biomed Eng., vol. 47, pp. 409-412, Mar. 2000.

[2] V. Vapnik, The Nature of Statistical Learning Theory. Berlin, Germany: Springer-Verlag, 1995.

[3] C. Cortes and V. Vapnik, "Support Vector Networks," Machine Learning, vol. 20, pp. 273-297, 1995.

[4] C. J. C. Burges, "A tutorial on support vector machines for pattern recognition," Data Mining Knowledge Discovery, vol. 2, no. 2, pp. 955-974, 1998.

[5] J. D. Z. Chen, Z. Y. Lin, and R. W. McCallum, "Abnormal gastric myoelectrical activity and delayed gastric emptying in patients with symptoms suggestive of gastroparesis," Dig. Dis. Sci., vol. 41, pp. 1538-1545, 1996.

[6] J. D. Z. Chen and R. W. McCallum, "EGG parameters and their clinical significance," in Electrogastrography: Principles and Applications. New York: Raven, 1994, pp. 45-73.

[7] R. Fletcher, Practical Methods of Optimization, 2 ed. New York: Wiley, 1987.

[8] B. Scholkopf, K. Sung, C. Burges, F. Girosi, P. Niyogi, T. Poggio, and V. Vapnik, "Comparing support vector machines with gaussian kernels to radial-basis function classifiers," IEEE Trans. Signal Processing, vol. 45, pp. 2758-2765, Nov. 1997.

[9] R. C. Eberhart and R. W. Dobbins, Neural Network PC Tools. San Diego: Academic, 1990.

[10] H. P. Parkman, A. D. Arthur, B. Krevsky, J. -L. C. Urbain, A. H. Maurer, and R. S. Fisher, "Gastroduodenal motility and dysmotility: An update on techniques available for evaluation," Amer. J. Gastroenterol., vol. 90, no. 6, pp. 869-892, 1995 .

[11] A. Dubois and M. Mizrahi, "Electrogastrography, gastric emptying, and gastric motility," in Electrogastrography: Principles and Applications. New York: Raven, 1994, pp. 247-256. 
[12] M. Hongo, Y. Okuno, N. Nishimura, T. Toyota, and S. Okuyama, "Electrogastrography for prediction of gastric emptying asate," in Electrogastrography: Principles and Applications. New York: Raven, 1994, pp. 257-269.

[13] T. L. Abell, M. Camilleri, V. S. Hench, and J. -R. Malagelada, "Gastric electromechanical function and gastric emptying in diabetic gastroparesis," Eur. J. Gastroenterol. Hepatol., vol. 3, pp. 163-167, 1991.

[14] K. L. Koch, R. M. Stern, W. R. Stewart, and M. W. Vasey, "gastric emptying and gastric myoelectrical activity in patients with diabetic gastroparesis: Effect of long-term domperidone treatment," Amer. J. Gastroenterol., vol. 84, pp. 1069-1075, 1989.

\section{Fast Detection and Characterization of Vessels in Very Large 3-D Data Sets Using Geometrical Moments}

\author{
Christine Toumoulin, Cezary Boldak, Jean Louis Dillenseger, \\ Jean Louis Coatrieux, and Yan Rolland
}

\begin{abstract}
An improved and very fast algorithm dealing with the extraction of vessels in three-dimensional imaging is described. The approach is based on geometrical moments and a local cylindrical approximation. A robust estimation of vessel and background intensity levels, position, orientation, and diameter of the vessels with adaptive control of key parameters, is provided during vessel tracking. Experimental results are presented for lower limb arteries in multidetector computed tomography scanner.
\end{abstract}

Index Terms-CTA, geometrical moment, three-dimensional segmentation.

\section{INTRODUCTION}

The ability to depict the vascular network is of critical importance for the diagnosis of vascular abnormalities, preoperative planning as well as preoperative actions. The recent advances provided by three-dimensional (3-D) magnetic resonance angiography (MRA) and multidetector computed tomography (CT) scanner [CTA] leads to new perspectives for objective and quantitative analysis of vascular networks as long as efficient algorithms are designed. These imaging modalities lead to very large data sets (several hundreds of megabytes) from which the vessels must be extracted under very strict time constraints to face the clinical requirements. Most of the current segmentation methods bring already relevant delineations of vessels but with too high computation time. They rely on intensity-based methods [1], generalized cylinder approximations [2], multiscale [3], [4] and skeletonization [5] schemes, and deformable model approaches [6] applied on successive two-dimensional (2-D) slices or on volume data.

The challenge is, therefore, to reach similar performance in few seconds on a standard PC platform. The present work is a step toward this objective. It relies on a moment-based algorithm [8] allowing, after interactive selection of 3-D seed points, to track the vessels and to locally estimate both the diameter and the orientation. Several improvements

Manuscript received December 2, 2000; revised January 8, 2001. Asterisk indicates corresponding author.

${ }^{*}$ C. Toumoulin is with Laboratoire Traitement du Signal et de l'Image, INSERM-Université de Rennes 1, Campus de Beaulieu, Bat 22, 35042 Rennes cedex (e-mail: christine.toumoulin@univ-rennes1.fr).

C. Boldak, J. L. Dillenseger, and J. L. Coatrieux are with Laboratoire Traitement du Signal et de l'Image, INSERM-Université de Rennes 1, Campus de Beaulieu, 35042 Rennes cedex.

Publisher Item Identifier S 0018-9294(01)03401-2. are brought including an efficient initialization, iterative positioning of the computation window, adaptive control of its size, and multibackground handling. These new features will be described after a short summary of the initial algorithm. Some preliminary results are then displayed on CTA data.

\section{METHOD}

Using the assumption that a vessel can be locally approximated by a cylinder, it has been shown in [8] that the 3-D geometrical moments of up to order two lead to analytical expressions of the orientation of the cylinder axis, the diameter when two homogeneous regions are considered, and the estimated mean gray levels inside and around the vessel. The discrete computation of these moments is performed on the set of elementary volumes (e.g., voxels) arranged on an isotropic grid using a spherical window, associated to a subvoxel decomposition. This way, the computation is reduced to mask convolutions. The tracking is performed by shifting the computation window according to the estimated orientation, and the stopping rule is stated as a decision between two hypotheses, presence or absence of the vessel. This solution has been validated on simulated data, for different signal to noise ratio and window sizes. As long as the window is larger than the vessel, diameter precision in the parameters estimation and robustness to noise have been demonstrated. The method was successfully applied to low field MRA data [8]. The core of the method remains unchanged and only the improvements will be reported here which allows to deal with some specific features of CT images, for instance the presence of more than two tissues.

\section{A. Algorithm Initialization}

In order to avoid the precise pointing of successive seed points located inside the several vascular branches of interest, a region is now defined by the user, in any of the three orthogonal cross sections. Maxima of intensities, corresponding to potential vessels, are then looked for in this region.

\section{B. Window Centering}

Either centered or noncentered moments can be used for the parameter computation but the estimations are better when using the former expressions. The orientation estimated at a given position of the window does not guarantee a precise positioning at the next point along the vessel, in particular when highly curved vessels are tracked. An iterative centering is, therefore, performed by computing the first order moments and then the other cylinder parameters are estimated.

\section{Window Size Adjustment}

The size of the vessels can widely vary (from tens to a very few voxels) when large segments of the body are explored or when diseases are concerned. In addition, the analytical diameter formulation as reported in [8], depends directly on the window size as well as the precision of the estimations mentioned above. The window size is then adjusted during the vessel tracking according to the previously computed width.

\section{Multiregion Handling}

In contrast with MRA, CT-based imaging modalities display other organs and tissues, and the enhanced vessels in CTA can be over or close to them, in other words, the computation window can include more than two regions. This problem is solved by exploring the vessel surroundings at each step along equally spaced directions in a plane 